\title{
An Agent-based Negotiation Model Selector for Managing Negotiation Processes in Corrective and Preventive Actions: A Conceptual Framework
}

\author{
Nur Huda,Jaafar ${ }^{1}$, Mohd Sharifuddin Ahmad ${ }^{2}$ \\ ${ }^{1}$ Faculty Of Computer and Mathematical Sciences ,Universiti Teknologi MARA, KM12, Jln Muar, 85009 \\ Segamat, Johor, Malaysia \\ 1nurhu378@johor.uitm.edu.my \\ ${ }^{2}$ College of Information Technology, Universiti Tenaga Nasional, Putrajaya Campus, JIn IKRAM-UNITEN, \\ 43000 Kajang, Selangor, Malaysia \\ ²sharif@uniten.edu.my
}

\begin{abstract}
Negotiation is an important process of management in any organizations especially if it involves quality management such as in corrective and preventive actions (CPA). The ability of software agent to implement automated negotiation can help organizations resolve or avoid conflicts. While many negotiation models have been developed, studies in intelligent selection of integrated negotiation models are still lacking. This paper proposes a conceptual framework for an agentbased negotiation model selector engine to resolve conflicts in the CPA activities. Several software agents will be involved to operate the proposed engine, in order to assist organizations in resolving conflicts and maintaining high quality management.
\end{abstract}

\section{Indexing terms/Keywords}

Negotiation; Software Agents; Corrective and Preventive Action; Selector Engine; Intelligent Systems

\section{Academic Discipline And Sub-Disciplines}

Software Agent, Quality Management

\section{SUBJECT CLASSIFICATION}

Artificial Intelligence Classification

TYPE (METHOD/APPROACH)

Conceptual Paper

\section{Council for Innovative Research}

Peer Review Research Publishing System

Journal: International Journal of Management \& Information Technology

Vol. 5, No. 2

editor@cirworld.com

www.cirworld.com, member.cirworld.com 


\section{INTRODUCTION}

An effective negotiation model and platform are important conditions for management in handling problems in their organizations. Management should look into the problems efficiently to avoid conflicts in the organization especially if the problems involve many parties. The clear information, processes and decision making are very important during the negotiation [1]. Nowadays, most projects or problems are solved by teamwork effort. Conflicts in teams should be reduced or completely avoided to achieve efficient negotiation result. Otherwise, the problems will not be completely resolved [2].

In quality management, one of the areas that needs conflict resolution via negotiation is corrective and preventive actions (CPA) enforcement. Without an effective negotiation process the CPA would fail to meet the quality management objectives. To maintain quality management in organizations, the CPA should be efficiently managed by a well-qualified team especially in the negotiation process.

Many technologies have been developed to implement automated negotiation. One of the famous technologies exploits the intelligent software agent paradigm. With three of its main capabilities: reactive, pro-active and social ability [3], a software agent can work on behalf of humans [4]. According to Wooldridge [3], reactive means the software agents are enabled to perceive their environment and responds to changes that occur in it. While proactive characterizes the agent to make attempts to achieve its goals by taking its own initiatives. With social ability, the agent has the capability to interact with other agents for achieving its goals. These capabilities would help the agent to negotiate on a user's behalf. Currently, various techniques are being used by researchers such as English auction, Dutch auction and games theory. These theories have been developed and commercialized in various fields such as enterprise management [5], scheduling [6], emarket [7] and manufacturing [8].

While there are research that exploit software agents in negotiation theories and models [9] [10] [11] [12] [13], it seems that there is a lack of studies on developing algorithms that select the best agent-based negotiation model for a particular organizational conflict. We hypothesize that the conception and implementation of agent-based negotiation model selector engine in resolving conflicts in CPA can assist organizations in effectively managing their quality initiatives. The relevance of this engine is justified due to the unpredictable conflicts that are experienced by organizations that require different negotiation approaches.

This paper proposes a conceptual framework of an agent-based negotiation model selector engine. We discuss a brief overview of the CPA, examples of negotiation problems in the CPA, the proposed conceptual framework for agent-based negotiation selector engine and future work in this paper.

\section{BRIEF OVERVIEW OF CORRECTIVE AND PREVENTIVE ACTIONS (CPA)}

Most organizations emphasize on continual improvement in order to ensure that the delivery of products or services achieves the quality standard. Continual improvement is important to maintain competitiveness with their rivals. According to Wahid and Corner [14], more emphasis must be placed on continual improvement and preventive actions during the maintenance stage of quality management. For this reason, corrective and preventive actions are crucial for top management and employees to review, especially when they want to plan out strategies for their businesses. Based on the study from Motchman and Moore [15], the corrective actions are only applied to correct the problems, while the preventive actions are taken to prevent the occurrence of a potential problems. During the enforcement of the CPA, the problems and the causes of problems that appear are identified. Based on the analysis, brainstorming sessions are conducted to identify the best solutions for the problems. The CPA activities continue non-stop until the solutions are implemented. The solution actions for the CPA are continuously observed and evaluated. At this stage, the commitment from top management and employees are fully needed.

Normally, the CPA processes involve a team that may consists of a group of staff from the same department or from different departments. Therefore, the delegation of tasks must be managed efficiently. During the task delegation process, negotiation activities ensue. While attempts are made to avoid conflicts during the negotiation process, conflicts do occur, jeopardizing the timely completion of the CPA.

\section{EXAMPLES OF NEGOTIATION PROBLEMS IN CORRECTIVE AND PREVENTIVE ACTIONS}

The CPA enforcement, which involves a large number of staff and departments, depends on the size of the problem. Consequently, teamwork is very important to minimize conflicts during problem-solving processes. If any conflicts arise while the CPA processes are underway and no steps are taken to address them, it may portray a negative image on good quality management and the organization in general. Below are examples of some conflicts that occur in an organization during the CPA enforcement:

\section{Case Study 1:}

Rooms belonging to department B are in department A. Occasionally, the corrective and preventive action (CPA) towards these rooms involve another department. The unclear information and direction may produce the conflict among the departments. This conflict may bring unhealthy working environment in organization. So, negotiation among these departments is very important to ensure the process of CPA runs smoothly. The roles of each department should be identified.

Case Study 2: 
The CPA activity involves many departments and a hired contractor to complete the activity that must complete on schedule. In this case, the department that looks after the contractor needs to be identified. The department-in-charge then mediates between the contractor and the relevant departments. The department-in-charge should inform any latest information to the relevant departments and the contractor as well. If the contractor fails to complete the work on time, the department-in-charge will call a meeting to negotiate among the departments.

\section{Case Study 3:}

The responsibility assigned to each staff is unbalanced. This difference in responsibility causes psychological dissatisfaction and affects jobs' performance. Consequently, this difference gives rise to uncooperative behavior among staff, subsequently jeopardizing the negotiation process. To resolve the problem of task delegation, the Head of department needs to intervene.

\section{Case Study 4:}

Staff do not receive the latest information while the CPA activity is underway. For example, staff $A$ needs information from staff $B$ to proceed with the next task. However, because of late delivery of information, staff B cannot complete the task on time.

The various types of conflicts in the CPA show that the organization needs a medium for solving these conflicts. However, the same approach cannot be used to resolve different conflicts. We propose an algorithm that reasons and selects the best negotiation model for resolving conflicts. By exploiting existing negotiation theories and using the capabilities of software agent, a proposed software agent-based negotiation selector engine may help organizations in managing the negotiation processes for the CPA enforcement.

\section{THE PROPOSED CONCEPTUAL FRAMEWORK}

The proposed software agent negotiation selector engine will be designed to implement the automated negotiation. According to Cao [16], the automated negotiation system consists of computational agents from various individuals or organizations that are capable in reaching an agreement through negotiation. In our proposed conceptual framework as shown in Figure 1, the automated negotiation is modeled and compiled from several existing negotiation theories (shown as Theory $\mathrm{A}-\mathrm{D})$.

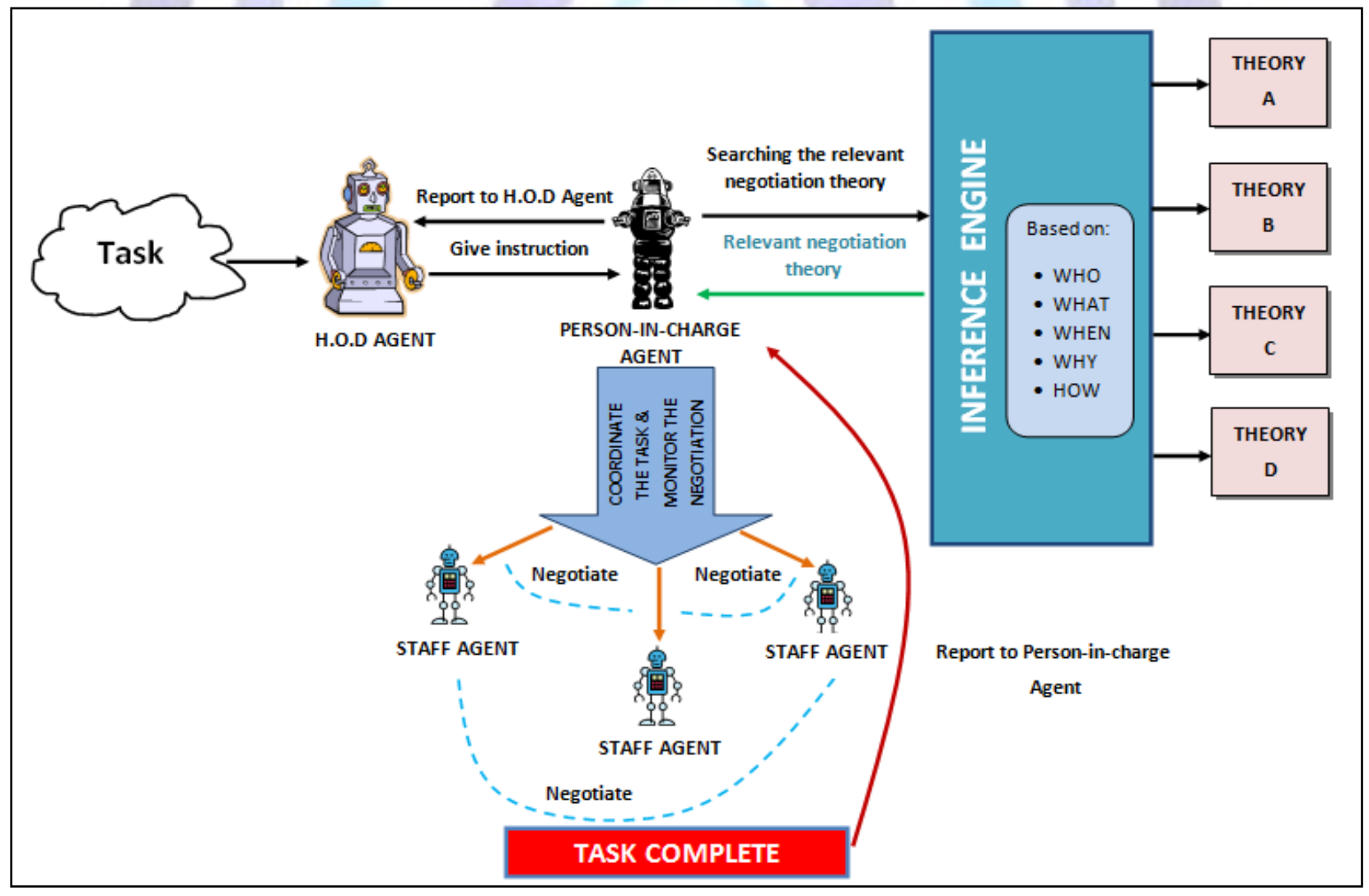

Figure 1: The Proposed Software Agent Negotiation Selector Engine

The engine start working as soon as the person-in-charge agent gets instructions from head of department (HOD) agent. Once the process starts, the person-in-charge agent looks for the relevant negotiation theory based on the task model. The inference engine is used to reason and identify the best theory to use. The search is based on who, what, when, why and how information model. The engine sets the priority of theory based on the given models. If the agent still faces the negotiation problem using the first suggested theory, it switches to another theory based on a priority scheme. 
Once the best negotiation theory is identified, the person-in-charge agent coordinates the task and proceeds the negotiation with the staff agents. It also monitors the negotiation process while it is underway. If the negotiation is successful and the task is completed, the staff agent reports the results to the person-in-charge agent. During the negotiation process, the interaction between agents can be either (i) one-to-one (ii) many-to-one or (iii) many-to-many negotiation.

\section{CONCLUSION AND FUTURE WORK}

While the framework looks somewhat trivial, several challenging issues are required to be investigated. To develop the selector engine, the negotiation protocol, the negotiation strategies and the agent's decision-making models are the areas that we need to investigate. Several theories in agent-based negotiation must also be studied and tested on the case studies. The criteria for setting the priority in choosing the best theory should be taken into account when developing this engine. These are areas that we shall look at in our future work.

\section{REFERENCES}

[1] I. S. Fulmer and B. Barry, "THE SMART NEGOTIATOR: COGNITIVE ABILITY AND EMOTIONAL INTELLIGENCE IN NEGOTIATION," The International Journal of Conflict Management, vol. 15, no. 3, pp. 245272, 2007.

[2] J. Liu, P. Fu, and S. Liu, "Conflicts in top management teams and team / firm outcomes approaches," International Journal of Conflict Management, vol. 20, no. 3, pp. 228-250, 2008.

[3] M. Wooldridge, An Introduction to Multi-agent Systems, First Edit. United Kingdom: John Wiley \& Sons Ltd, 2009, p. 461.

[4] V. R. Lesser, "Cooperative Multiagent Systems: A Personal View of the State of the Art," IEEE Transactions on Knowledge and Data Engineering, vol. 11, no. 1, pp. 133-142, 1999.

[5] T. Kaihara and S. Fujii, "Game theoretic enterprise management in industrial collaborative networks with multiagent systems," International Journal of Production Research, vol. 46, no. 5, pp. 1297-1313, Mar. 2008.

[6] C. J. Huang and L. M. Liao, "An agent-based dynamic scheduling for parallel machine under machine eligibility constraints," in Management of Innovation and Technology (ICMIT), 2012 IEEE International Conference on, 2012, pp. 689-693.

[7] S. Adabi, A. Movaghar, A. M. Rahmani, and H. Beigy, "Negotiation strategies considering market, time and behavior functions for resource allocation in computational grid," The Journal of Supercomputing, Jul. 2012.

[8] C. J. Anumba, Z. Ren, A. Thorpe, O. O. Ugwu, and L. Newnham, "Negotiation within a multi-agent system for the collaborative design of light industrial buildings," Advances in Engineering Software, vol. 34, no. 7, pp. 389-401, Jul. 2003.

[9] M. Cao, "Goal deliberated agent architecture for multi-strategy selection in automated negotiation," in Proceedings of the 14th Annual International Conference on Electronic Commerce - ICEC '12, 2012, pp. 159-160.

[10] S. Heras, J. Jordán, V. Botti, and V. Julián, "Argue to agree: A case-based argumentation approach," in International Journal of Approximate Reasoning, 2013, vol. 54, no. 1, pp. 82-108.

[11] H. J. Heppner, T. Bertsch, B. Alber, a S. Esslinger, C. Dragonas, J. M. Bauer, and C. C. Sieber, "Procalcitonin: inflammatory biomarker for assessing the severity of community-acquired pneumonia--a clinical observation in geriatric patients.," Gerontology, vol. 56, no. 4, pp. 385-9, Jan. 2010.

[12] S. Ilie, C. Bădică, A. Bădică, L. Sandu, R. Sbora, M. Ganzha, and M. Paprzycki, "Information flow in a distributed agent-based online auction system," in Proceedings of the 2nd International Conference on Web Intelligence, Mining and Semantics - WIMS '12, 2012, p. 1.

[13] M. Navarro, S. Heras, V. Botti, and V. Julián, "Towards real-time agreements," Expert Systems with Applications, vol. 40, no. 10, pp. 3906-3917, Aug. 2013.

[14] R. A. Wahid and J. Corner, "Critical success factors and problems in ISO 9000 maintenance," International Journal of Quality \& Reliability Management, vol. 26, no. 9, pp. 881-893, 2009.

[15] T. L. Motschman and S. B. Moore, "Corrective and preventive action.," Transfusion science, vol. 21, no. 2, pp. 163-78, Oct. 1999.

[16] M. Cao, "Multi-strategy Selection Supported Automated Negotiation System Based on BDI Agent," in 2012 45th Hawaii International Conference on System Sciences, 2012, pp. 638-647.

\section{Author' biography with Photo}

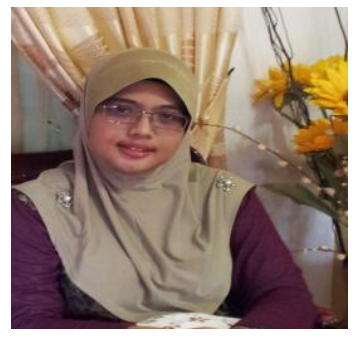

Nur Huda Jaafar is a lecturer at the MARA University of Technology, Malaysia in Computer Science. She obtained her Master degree from the MARA University of Technology in 2006. Her special interest is in computer science education and software agents. Besides involving in innovation research in computer science, she also committed actively in quality management at the higher education institution. Currently, she is doing her Ph.D, under the supervision of Associate Professor Mohd Sharifuddin Ahmad, focusing on modeling the automated negotiation strategy framework for software agents in managing corrective and preventive action. 


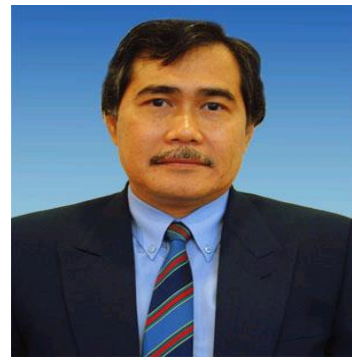

Mohd Sharifuddin Ahmad received his B.Sc. in Electrical and Electronic Engineering from Brighton Polytechnic, UK in 1980. He started his career as a power plant engineer specialising in Process Control and Instrumentation in 1980. After completing his MSc in Artificial Intelligence from Cranfield University, UK in 1995, he joined UNITEN as a Principal Lecturer and Head of Dept. of Computer Science and Information Technology. He obtained his Ph.D from Imperial College, London, UK in 2005. He has been an Associate Professor at UNITEN since 2006. His research interests includes applying constraints to develop collaborative frameworks in multi-agent systems, collaborative interactions in multi-agent systems and tacit knowledge management using Al techniques. 